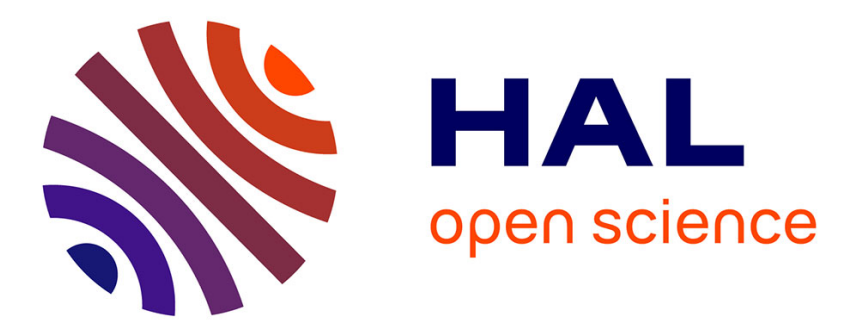

\title{
La basilique funéraire de la rue Malaval à Marseille (Bouches-du-Rhône)
}

\author{
Manuel Moliner
}

\section{To cite this version:}

Manuel Moliner. La basilique funéraire de la rue Malaval à Marseille (Bouches-du-Rhône). Gallia - Archéologie de la France antique, 2006, Antiquité tardive, haut Moyen Âge et premiers temps chrétiens en Gaule méridionale (première partie) : réseau des cités, monde urbain et monde des morts, 63, pp.131-136. 10.3406/galia.2006.3292 . hal-01914327

\section{HAL Id: hal-01914327 \\ https://hal.science/hal-01914327}

Submitted on 8 Jan 2020

HAL is a multi-disciplinary open access archive for the deposit and dissemination of scientific research documents, whether they are published or not. The documents may come from teaching and research institutions in France or abroad, or from public or private research centers.
L'archive ouverte pluridisciplinaire HAL, est destinée au dépôt et à la diffusion de documents scientifiques de niveau recherche, publiés ou non, émanant des établissements d'enseignement et de recherche français ou étrangers, des laboratoires publics ou privés.

\section{(ㅇ)(1) $\$$}

Distributed under a Creative Commons Attribution - NonCommercial - NoDerivatives| 4.0 


\title{
LA BASILIQUE FUNÉRAIRE DE LA RUE MALAVAL À MARSEILLE (BOUCHES-DU-RHÔNE)
}

\author{
Manuel MOLINER
}

\begin{abstract}
Mots-clés. Église funéraire, nécropole, sarcophage, memoria, sépulture privilégiée, dispositifs liturgiques, autel, reliquaire, épigraphie.

Résumé. Des fouilles archéologiques préventives, réalisées en 2003-2004, ont révélé des vestiges inédits d'une église paléochrétienne et d'une importante nécropole au nord immédiat de la ville antique de Marseille.

$A u V^{e}$ s., un bâtiment de plan basilical orienté, doté d'une grande abside, est construit le long de l'ancienne voie antique. Épierré sans doute au VII s., cet édifice recèle dans le chœur une imposante tumulatio ad sanctos de sarcophages autour d'une sépulture vénérée. Celle-ci comporte des dispositifs liturgiques particuliers liés à la vénération de deux personnages anonymes. Sous l'autel se trouvait un caisson à reliques également associé à des actes liturgiques. À l'intérieur et hors l'église s'est développée une zone sépulcrale dense de plus de 200 tombes aux architectures funéraires sobres mais variées destinées à des hommes, des femmes et des enfants. Le mobilier funéraire est rare, quelques épitaphes sur marbre sont à signaler. Des éléments lapidaires témoignent de décors simples. Le vocable de cette église contemporaine du premier édifice de Saint-Victor est inconnu. Des recherches scientifiques sont en cours, parallèlement à un programme de reconstitution à l'identique du site.
\end{abstract}

Key-words. Funerary church, cemetery, sarcophagus, memoria, burial, liturgical devices, altar, reliquary, epigraphy.

Abstract. In 2003-2004, unknown remains of an early Christian church and of a vast cemetery have been uncovered during archaeological rescue excavations, nearby the ancient town of Marseille.

During the $5^{\text {th }}$ c., a basilical shape monument, positioned with a wide apse, is built along the Roman road. The choir of this building - its stones probably have been removed in the $7^{\text {th }} c$. - contains an imposing tumulatio ad sanctos of sarcophages around a venerated burial. This tomb is equipped with particular liturgical devices in connection with the veneration of two anonymous individuals. Underneath the altar, a case with relics, being also associated to liturgical gestures. Inside and outside the church a sepulchral area of high density contains more than 200 burials with sober but varied funeral features, for men, women and children. Grave-goods are scarce outside some marble funerary inscriptions. Simple designs are visible on stone pieces. We don't know to whom this church, contemporary to the first monument of Saint-Victor, was dedicated. Scientific research is going on into a reconstruction programme.

Translation: Isabelle FAUDUET

Schlagwörter. Grabkirche, Nekropole, Sarkophag, memoria, hervorgehobene Bestattung, liturgische Vorrichtungen, Altar, Reliquiar, Epigraphik.

Zusammenfassung. In den Jahren 2003-2004 durchgeführte Präventivgrabungen haben unmittelbar nördlich des antiken Marseille zur Aufdeckung unbekannter Überreste eines frühchristlichen Kirchenbaus und einer bedeutenden Nekropole geführt.

Entlang der antiken Straße wurde im 5. Jahrhundert eine Gebäude mit basilikalem Grundriß und großer Apsis errichtet. Der Bau, der zweifellos bereits im 7. Jahrhundert dem Steinraub zum Opfer fiel, barg in seinem Chorraum eine eindrucksvolle tumulatio ad sanctos von Sarkophagen um eine verehrte Grabstätte herum. Dieses Grab weist spezifische liturgische Vorrichtungen auf, die mit der Verehrung zweier unbekannter Personen in Verbindung zu bringen sind. Unter dem Altar befand sich ein gleichfalls mit liturgischen Handlungen zusammenhängender Reliquienbehälter. In und außerhalb der Kirche entwickelte sich ein dichtes Gräberfeld mit mehr als 200 Bestattungen, mit schlichtem aber für Männer, Frauen und Kinder unterschiedlich angelegtem Grabbau. Beigabenausstattung ist selten; lediglich einige Marmorepitaphien sind anzuzeigen. Die steinernen Bauteile weisen nur einfache Verzierungen auf. Das Patrozinium dieser Kirche die mit dem ersten Bau von Saint Victor gleichzeitig ist, ist nicht bekannt. Parallel zu dem Projekt einer originalgetreuen Rekonstruktion des Platzes sind derzeit weitere wissenschaftliche Untersuchungen im Gang.

Übersetzung: Stefan WIRTH 
Les vestiges inédits d'une église funéraire suburbaine et ceux, pressentis, de la nécropole dite du Lazaret, signalée $\mathrm{au} \mathrm{XIX}^{\mathrm{e}} \mathrm{s}$., ont été retrouvés à l'occasion des travaux d'un parking souterrain entre juillet 2003 et mars 2004 à Marseille, dans le quartier de la Joliette (fig. 64). Les fouilles archéologiques ont été menées par une équipe de l'Institut national de recherches en archéologie préventive (INRAP), antenne Méditerranée, dirigée par M. Moliner, archéologue municipal de la ville de Marseille, sur prescription du Service régional de l'archéologie de Provence-Alpes-Côte d'Azur.

La première occupation du site se limite à deux portions de murs, une canalisation en amphores hispaniques et du mobilier appartenant à un monument d'époque impériale, sans doute à vocation funéraire. Retrouvés à la limite orientale de la fouille, ces vestiges ténus sont les ultimes témoins de la nécropole qui se développait le long de la voie antique conduisant à Aix à partir de la porte nord de la cité. Ce territoire réservé aux morts a été détruit, sur la zone étudiée, par l'installation du bâtiment chrétien au $\mathrm{V}^{\mathrm{e}} \mathrm{s}$. Le Bas-Empire n'est pas attesté sur le site.

Les larges fondations de l'édifice paléochrétien ont été retrouvées en dépit d'épierrements importants, sans doute effectués à partir du VII ${ }^{\mathrm{e}}$ s., qui ont fait disparaître toutes les élévations. L'église est orientée. Le plan de type basilical, incomplet, consiste en une nef unique de plus de $30 \mathrm{~m}$ de long pour $16 \mathrm{~m}$ de large dans œuvre; elle est dotée d'une abside semi-circulaire à l'est, large de $12 \mathrm{~m}$ et profonde de 6,60 m. Les murs sud et ouest sont situés en dehors des limites d'investigation. L'entrée principale n'est pas connue, elle est peut-être à localiser dans la façade ouest située hors fouille. En revanche, une petite porte est attestée sur le flanc nord du mur d'abside : s'agit-il d'un accès réservé ? Ce passage donne sur la voie par un escalier composé de blocs décorés provenant d'un mausolée antique en remploi. Si une charpente de bois est tout à fait probable pour la couverture de la nef, aucun élément ne permet de restituer, dans l'état actuel des recherches, la couverture du chevet : voûte, demicoupole ou toiture à pans coupés ? La nature des sols nous échappe, sauf pour l'état primitif dans le chœur, où un sol en béton de tuileau maigre est attesté.

$\mathrm{Au}$ centre du chœur, l'autel a été identifié à partir d'éléments architecturaux in situ (fig. 65). Une base en marbre avec encoches pour cinq piliers, support d'une table d'autel disparue, protégeait le caisson des reliques, un bloc de calcaire antique retaillé (fig. 66). Les deux moitiés d'une base d'autel primitive en calcaire rose du Cap-Couronne (Martigues) ont été réutilisées pour le second état de l'autel qui comportait une base en marbre. La base primitive présente quatre encoches pour le support de la table d'autel et deux percements particuliers au centre. Ces derniers éclairent de manière inédite les pratiques liturgiques abandonnées dans le second état. En effet, un trou circulaire servait au versement d'un liquide sous l'autel, dans le caisson des reliques composé de deux compartiments, et un second orifice, plus large, était utilisé pour permettre la sanctification d'objets par le contact des saintes reliques. Celles-ci n'ont pas été retrouvées car elles ont été déplacées dans l'Antiquité tout comme, selon toute vraisemblance, la table d'autel probablement en marbre.

Entre l'autel et la porte latérale du mur d'abside, une sépulture privilégiée (memoria) a été installée dès l'origine. Tout autour de cette dernière s'est organisée une tumulatio ad sanctos caractérisée par une presse de sarcophages inviolés dans le chœur (fig. 67). Cette ferveur se retrouve dans les ensevelissements en grand nombre tant à l'intérieur de l'église qu'à l'extérieur. Cette tombe remarquable se caractérisait par un aménagement monumental en élévation dans le chœur. Neuf plaques de marbres polychromes, maintenues par quatre piliers de marbre blanc, composaient un décor sobre mais bien en évidence. Les deux chancels sud et ouest, en marbre blanc monolithe, comportaient un décor géométrique d'écailles imbriquées d'exécution délicate offert à la vue des fidèles. Ce décor ornait deux tombes jumelles accolées en sarcophage de calcaire rose de grandes dimensions. Le dallage de couverture, formé de plaques de marbre gris ou blanc, était percé en deux points par des orifices circulaires destinés à l'introduction d'un liquide. Un tuyau de bronze, reste d'un entonnoir, traversait les couvercles de calcaire et permettait le versement d'un liquide (de l'huile ?) dans la tombe (fig. 68). Ce liquide acquérait des vertus particulières par le contact avec le cercueil en plomb qui protégeait la dépouille. Enfin, un exutoire en bronze, placé à l'arrière de la sépulture, permettait de recueillir ce liquide dans des ampoules de verre destinées probablement à des eulogies. Les études pluridisciplinaires engagées permettront-t-elles d'envisager un procédé de sanctification? À l'instar de l'abandon de certains gestes dans le second état de l'autel, il ne semble plus y avoir de récupération du précieux liquide lorsque le sol de l'abside a été exhaussé. En revanche, si la tombe double semble avoir été toujours respectée et vénérée, nous ignorons quels gestes étaient alors pratiqués. L'étude en cours des restes humains de cette dernière a révélé la présence de deux individus masculins âgés, sans aucun apparat, mais des restes de tissus (vêtements?) ont été repérés sur ces corps anonymes. 


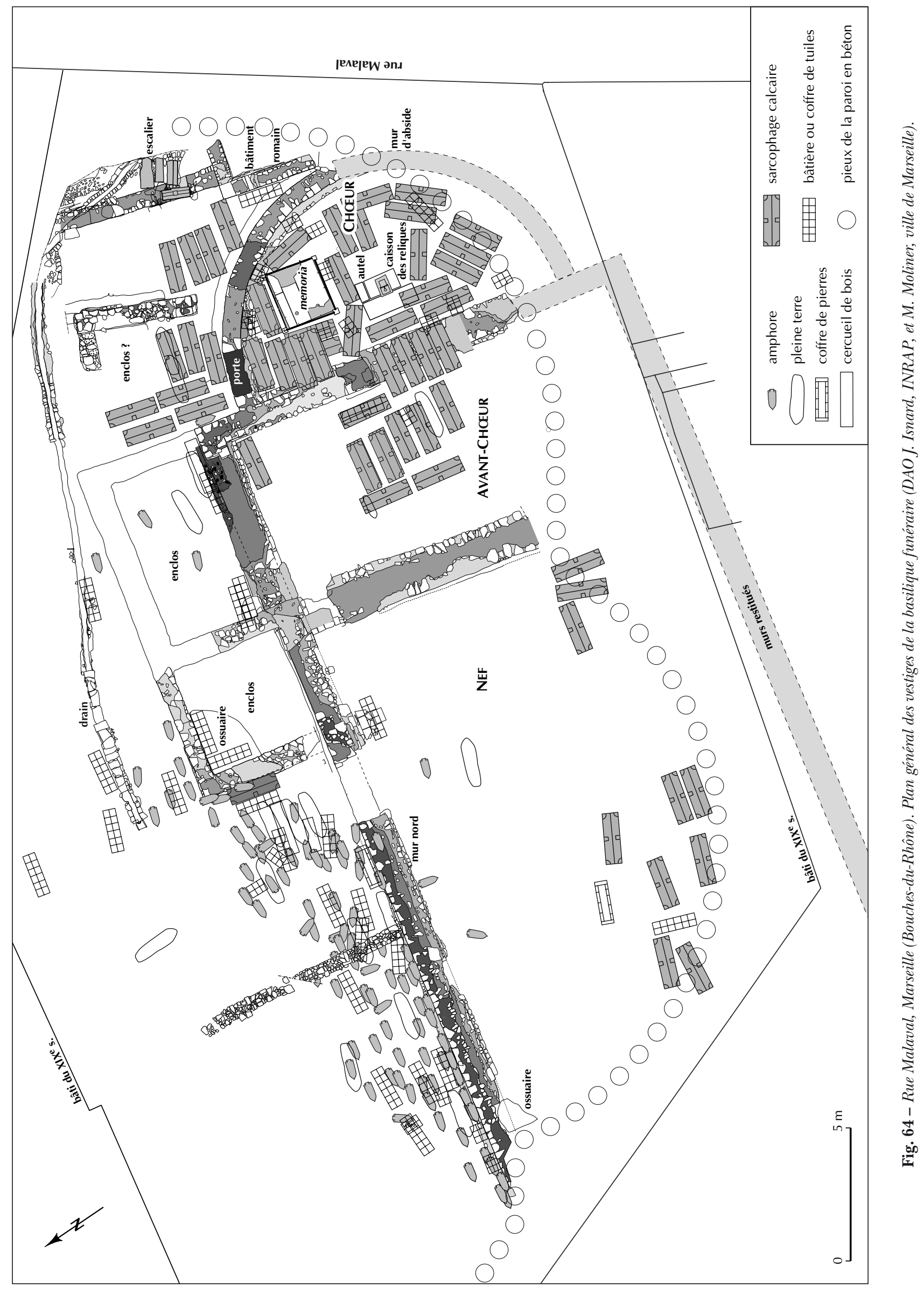




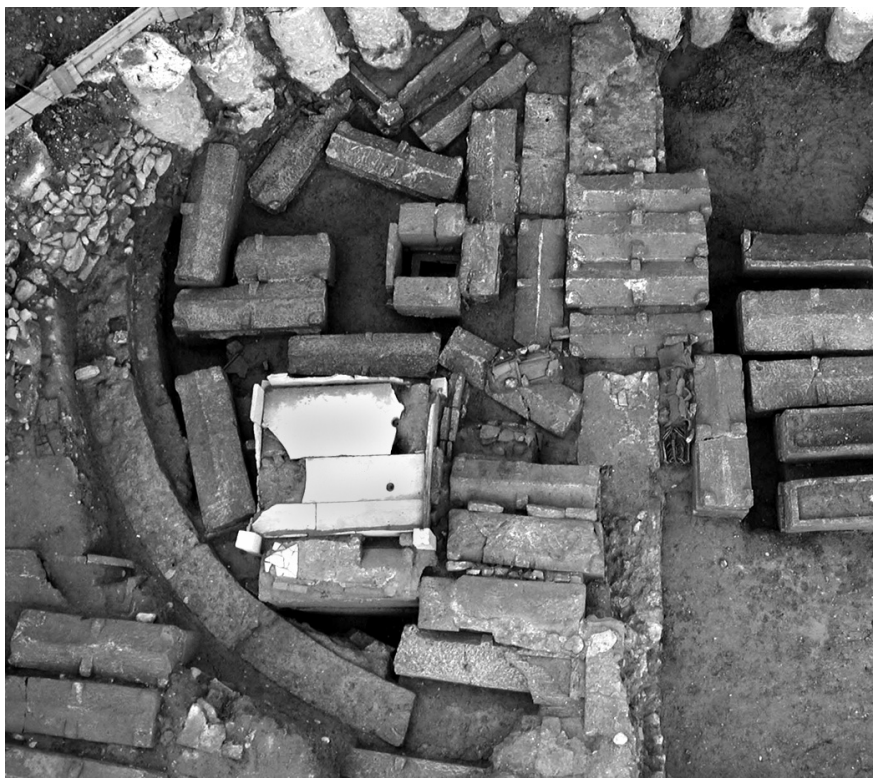

Fig. 65 - Rue Malaval, Marseille (Bouches-du-Rhône). Vue générale du chœur de la basilique funéraire; le nord se trouve dans la partie inférieure de la photo (cliché M. Moliner, ville de Marseille).

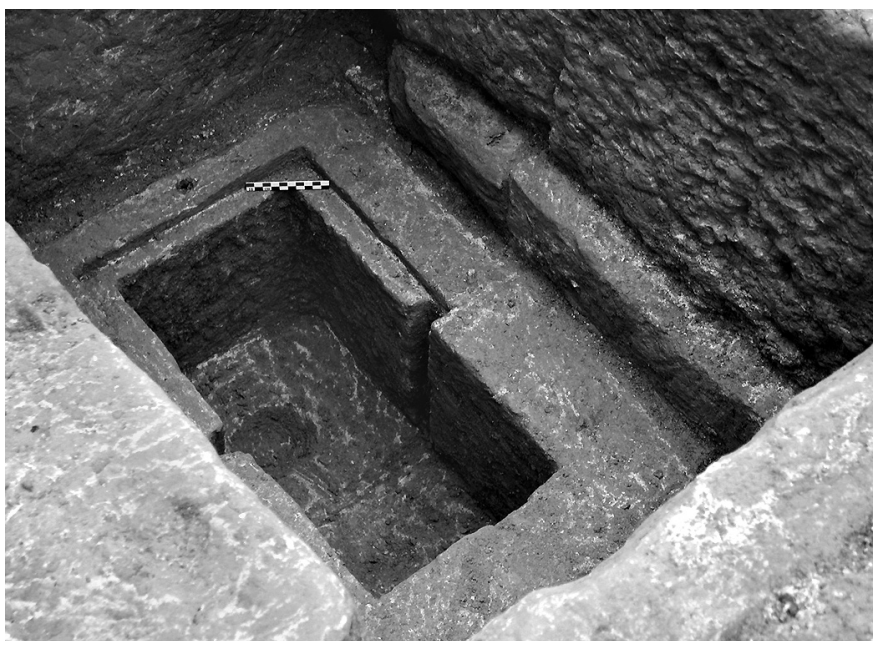

Fig. 66 - Rue Malaval, Marseille (Bouches-du-Rhône). Basilique funéraire: le caisson des reliques en calcaire rose (cliché M. Moliner, ville de Marseille).

Une vaste nécropole s'est développée autour de cette sépulture vénérée, dans le chœur, dans la nef et audehors de l'église. L'étude archéoanthropologique en cours est conduite par A. Richier et R. Lisfranc (INRAP). 228 tombes ont été reconnues, qui documentent la mise en place, le développement et l'abandon de cette aire sépulcrale au sein de laquelle la grande majorité des individus

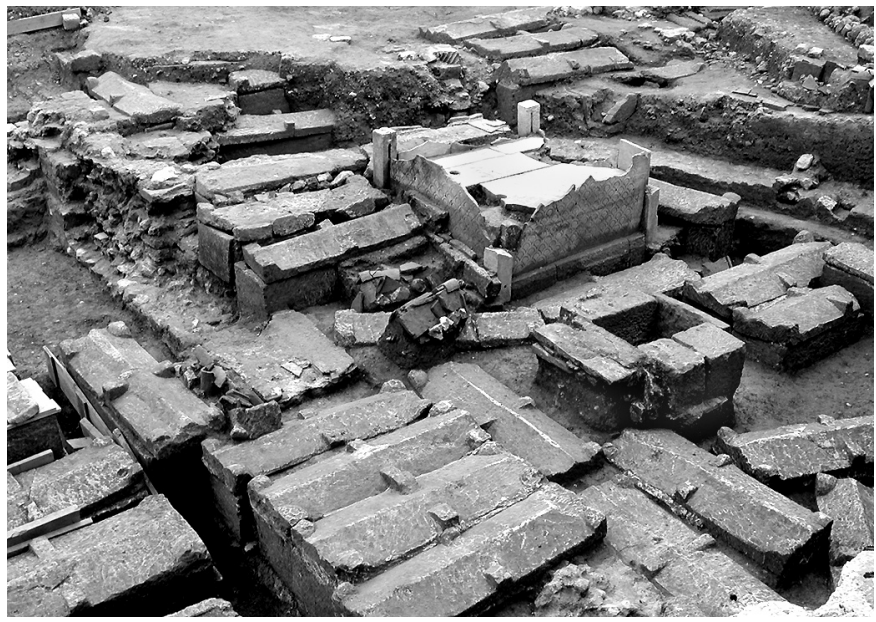

Fig. 67 - Rue Malaval, Marseille (Bouches-du-Rhône). Basilique funéraire : vue d'ensemble de la tombe privilégiée et de la presse des sarcophages prise du sud-ouest (cliché M. Moliner, ville de Marseille).

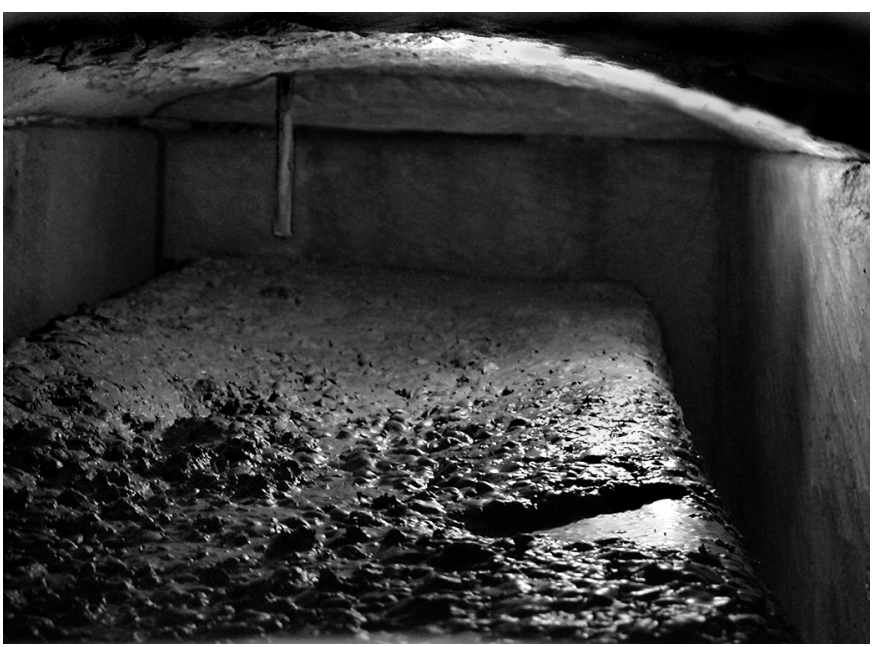

Fig. 68 - Rue Malaval, Marseille (Bouches-du-Rhône). Basilique funéraire: un des deux tuyaux en bronze pénétrant dans la tombe vénérée au-dessus du cercueil de plomb (cliché M. Moliner, ville de Marseille).

est enterrée tête à l'ouest. Une soixantaine de sarcophages ont été dégagés, essentiellement dans l'église et contre le chevet nord. Des tombes en pleine terre, souvent avec cercueils de bois, et une quarantaine de tombes sous tuiles, en caissons ou bâtière, ont été fouillées à l'extérieur de l'édifice. On signalera un groupe particulier de petites bâtières pour enfants inhumés dans le chøur. Enfin, une 


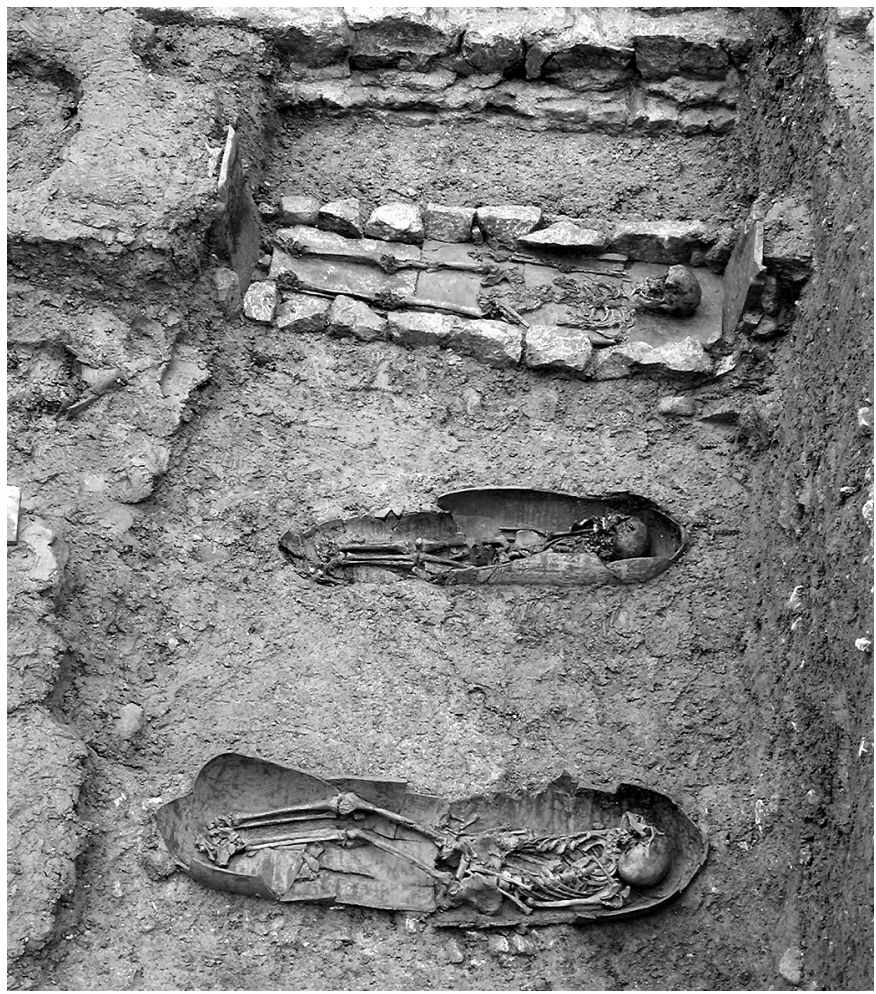

Fig. 69 - Rue Malaval, Marseille (Bouches-du-Rhône). Basilique funéraire: sépultures en amphores et bâtières en cours de fouille; les défunts en décubitus dorsal sont inhumés la tête à l'ouest (cliché M. Moliner, ville de Marseille).

centaine de tombes en amphores africaines, orientales et hispaniques, essentiellement pour des enfants - et quelques jeunes adultes - ont été retrouvées en quasitotalité à l'extérieur du bâtiment (fig. 69 et 70). Des enclos funéraires ont été identifiés à l'extérieur de l'église. L'étude de cette population fait apparaître des adultes de tout âge, hommes et femmes, des enfants et des nourrissons. La réouverture des sarcophages pour des inhumations successives et des réductions de corps, ainsi que la présence d'un important ossuaire probablement constitué de curage de sarcophages, restituent un groupe humain de plus de 300 individus en dépit de la destruction de tombes lors de l'épierrement de l'édifice. La stratigraphie des mises en terre et l'étude typologique des réceptacles, en particulier du matériel amphorique, placent l'occupation funéraire aux $\mathrm{V}^{\mathrm{e}}-\mathrm{VI}^{\mathrm{e}}$ s. Le mobilier accompagnant le défunt est pratiquement inexistant : seuls des éléments d'habillement ou de parure très simples sont attestés, ainsi que de très rares offrandes, mais uniquement dans les toutes premières sépultures (céramique, verrerie). Un lot important de

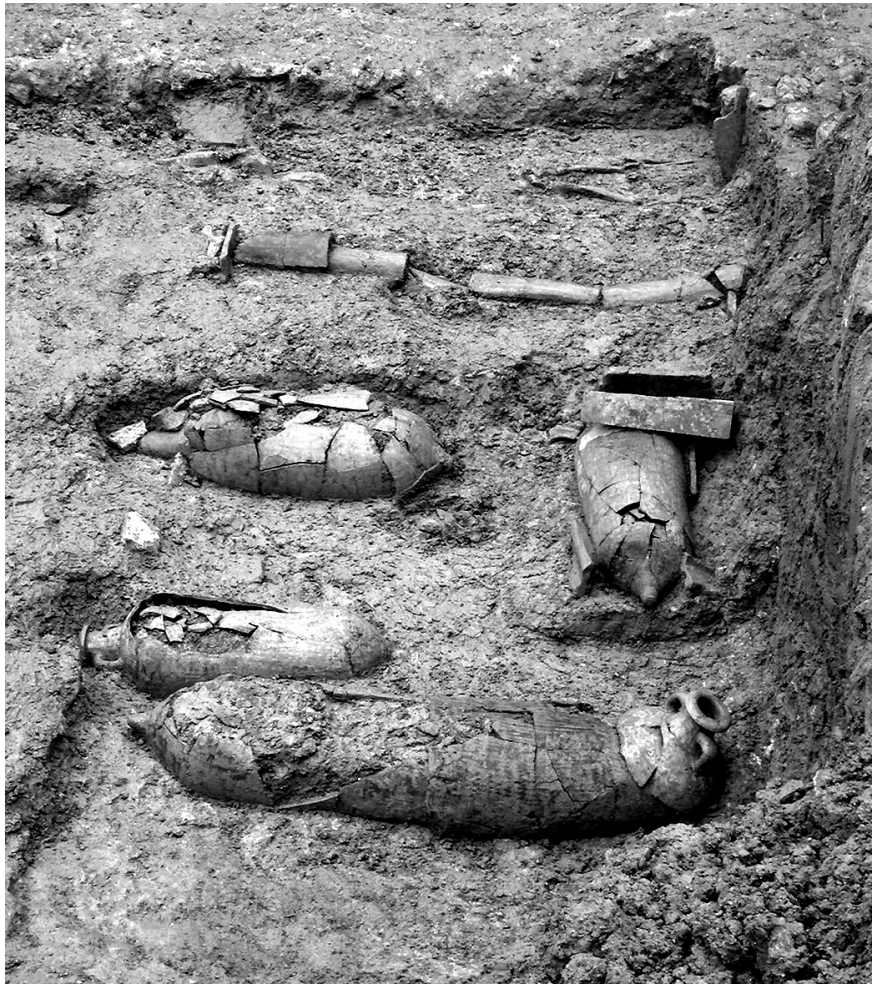

Fig. 70 - Rue Malaval, Marseille (Bouches-du-Rhône). Basilique funéraire: sépultures en amphores et bâtières contre le mur nord de l'église au moment de leur mise au jour (cliché M. Moliner, ville de Marseille).

petit monnayage de bronze a été retrouvé en stratigraphie auprès de la tombe privilégiée et des reliques. Quelques fragments d'inscriptions sur marbre et l'épitaphe complète d'un jeune homme nommé Stephanus révèlent l'identité de certains défunts. Le vocable de l'église n'est pas attesté par l'archéologie, peut-être s'agit-il de la basilica sancti Stephani qu'un texte de Grégoire de Tours décrit comme étant «très proche de la ville ».

La basilique de la rue Malaval et ses inhumations sont sans doute à l'origine de la nécropole nord de la Marseille chrétienne, repérée au XIX ${ }^{\mathrm{e}} \mathrm{s}$. La nécropole du Lazaret est en effet signalée par des trouvailles répétées qui en attestent l'extension le long de la via Aquensis et par une magnifique maquette de 1864 (fig. 71).

Les vestiges de la basilique et son mobilier ont fait l'objet d'un programme de sauvegarde pour leur déplacement sur un autre site et une reconstitution à l'identique, avec les éléments originaux. Pour ce projet, en cours d'étude, les sarcophages du chœur et un certain nombre de sépultures ont été transportés dans le dépôt archéo- 


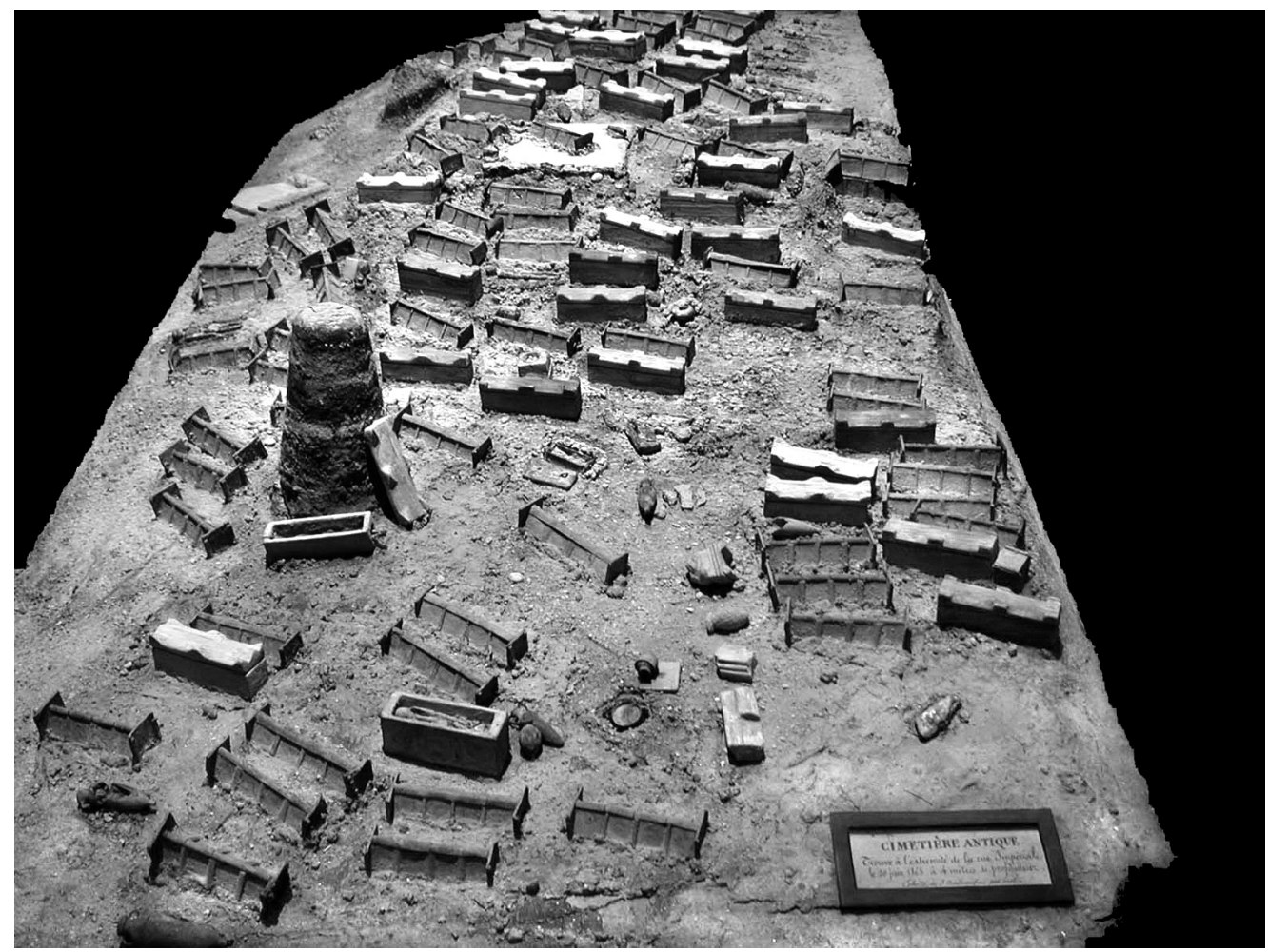

Fig. 71 - Marseille (Bouches-du-Rhône). Maquette de la nécropole du Lazaret par H. Augier en 1864 (cliché M. Moliner, ville de Marseille).

logique municipal, tout comme les éléments constitutifs de la tombe principale, dont les chancels décorés ont été consolidés. Les murs, quant à eux, ont fait l'objet de moulages.

La découverte d'une église funéraire inédite à Marseille, située au nord de la ville, telle une réplique de Saint-Victor sur la rive sud du port, mais surtout les témoignages uniques de pratiques liturgiques inconnues jusque-là sur le plan archéologique permettent de considérer ce gisement comme exceptionnel pour l'archéologie chrétienne bien au-delà de Marseille et de la région provençale. 